\title{
Mean level signal crossing rate for an arbitrary stochastic process
}

\author{
Yura, Harold T.; Hanson, Steen Grüner
}

Published in:

Optical Society of America. Journal A: Optics, Image Science, and Vision

Link to article, DOI:

10.1364/JOSAA.27.000797

Publication date:

2010

Document Version

Publisher's PDF, also known as Version of record

Link back to DTU Orbit

Citation (APA):

Yura, H. T., \& Hanson, S. G. (2010). Mean level signal crossing rate for an arbitrary stochastic process. Optical Society of America. Journal A: Optics, Image Science, and Vision, 27(4), 797-807.

https://doi.org/10.1364/JOSAA.27.000797

\section{General rights}

Copyright and moral rights for the publications made accessible in the public portal are retained by the authors and/or other copyright owners and it is a condition of accessing publications that users recognise and abide by the legal requirements associated with these rights.

- Users may download and print one copy of any publication from the public portal for the purpose of private study or research.

- You may not further distribute the material or use it for any profit-making activity or commercial gain

- You may freely distribute the URL identifying the publication in the public portal

If you believe that this document breaches copyright please contact us providing details, and we will remove access to the work immediately and investigate your claim. 


\title{
Mean level signal crossing rate for an arbitrary stochastic process
}

\author{
Harold T. Yura ${ }^{1}$ and Steen G. Hanson ${ }^{2, *}$ \\ ${ }^{1}$ Electronics and Photonics Laboratory, The Aerospace Corporation, Los Angeles, California 90009, USA \\ ${ }^{2}$ DTU Fotonik, Department of Photonics Engineering, Danish Technical University, \\ 4000 Roskilde, Denmark \\ *Corresponding author: Steen.Hanson@fotonik.dtu.dk
}

Received November 4, 2009; accepted January 6, 2010;

posted January 20, 2010 (Doc. ID 119557); published March 24, 2010

\begin{abstract}
The issue of the mean signal level crossing rate for various probability density functions with primary relevance for optics is discussed based on a new analytical method. This method relies on a unique transformation that transforms the probability distribution under investigation into a normal probability distribution, for which the distribution of mean level crossings is known. In general, the analytical results for the mean level crossing rate are supported and confirmed by numerical simulations. In particular, we illustrate the present method by presenting analytic expressions for the mean level crossing rate for various probability distributions, including ones that previously were unavailable, such as the uniform, the so-called gamma-gamma, and the Rice-Nakagami distribution. However, in a limited number of cases the present results differ somewhat from the result reported in the literature. At present, this discrepancy remains unexplained and is laid open for future discussion. (C) 2010 Optical Society of America
\end{abstract}

OCIS codes: $030.1630,030.1670,030.6140,030.6600$.

\section{INTRODUCTION}

The investigation of fade and surge statistics and the associated mean level crossing rate of a stationary differentiable random process has been of considerable interest since the pioneering work of Rice [1]. Physically, the derivative of each realization of such a random process is the derivative in the usual sense of the corresponding realization. For example, in optical communication systems, and especially for propagation through the atmosphere of information-carrying laser beams, the received light will be aberrated both by optical turbulence between the transmitter and the receiver and by line-of-sight platform jitter. This will inevitably result in intensity modulation of the received signal, the extent of which will be determined by the intermediate atmosphere and the optical setup. Thus, knowledge of fading and level crossings of the received, perturbed signal becomes of uttermost importance. In case of sensor systems, tracking of speckle patterns, shearing of dynamic speckle patterns, and derivation of the structure of a fringe pattern are commonly appearing issues of importance for the sensor systems. In these cases, the random nature of the underlying speckle structure will inevitably cause signal drop-out, the extent of which will constitute a major factor in the reliability of the sensor system. Thus, knowledge about the level crossings is of crucial importance in cases where the intensity variations possess statistical properties governed by random polarization and where coherent and incoherent addition random electromagnetic fields are interfered.

According to Rice, the mean level crossing rate (for both positive and negative crossings), $\nu\left(x_{0}\right)$, for an arbitrary stationary differentiable random process is given by

$$
\nu\left(x_{0}\right)=\int_{-\infty}^{\infty}|\dot{x}| p_{X, X}\left(x_{0}, \dot{x}\right) d \dot{x}
$$

where $\dot{x}$ is the first derivative of $x$ with respect to time (i.e., $d x / d t)$ and $p\left(x_{0}, \dot{x}\right)$ is the joint probability density function (PDF) of $x(t)$ and $\dot{x}(t)$ at time $t$. The subscripts in Eq. (1) refer to the arbitrary random process, while lowercase variables refer to the random variable. For a normal random process, where the joint PDF of $x(t)$ and $\dot{x}(t)$ are known to be independent [i.e., $p\left(x_{0}, \dot{x}\right)=p\left(x_{0}\right) p(\dot{x})$ ], Eq. (1) yields [2]

$$
\nu\left(x_{0}\right)=\frac{\sqrt{\sigma_{\dot{x}}^{2}}}{\pi} \exp \left(-\frac{\left(x_{0}-\mu\right)^{2}}{2 \sigma^{2}}\right)=\frac{\sqrt{-\ddot{R}(0)}}{\pi} \exp \left(-\frac{\left(x_{0}-\mu\right)^{2}}{2 \sigma^{2}}\right),
$$

where $\sigma_{\dot{x}}^{2}$ is the variance of the time derivative of the normal process, $\mu$ and $\sigma$ are the mean and standard deviation of the normal process, and $R(t)$ is the corresponding temporal autocorrelation coefficient.

Although analytic expressions for the mean level crossing rate $\nu\left(x_{0}\right)$ at an arbitrary level $x_{0}$ have been obtained for a limited number of other random processes, the corresponding analytic expressions for the mean level crossing rate for an arbitrary random process has remained elusive [3-6]. This is because the joint PDF of the level and its time derivative for an arbitrary stochastic process has not been obtained, and thus the integral in Eq. (1) cannot be determined. 
Khimenko [3] was the first to obtain analytic solutions of Eq. (1) for processes with statistically independent derivatives and corresponding processes that are functional transformations of these processes. For example, his results include processes with an exponential, Rayleigh, gamma, Maxwell, and Laplace distribution. Subsequently, several other authors have independently derived several of Khimenko's results including the exponential distribution, as the level-crossing rate of the intensity of speckle patterns have been the subject of both theoretical [7-10] and experimental [11,12] interest over the past several years. Barakat [4], based on the gamma distribution, obtained analytic results for the mean level crossing rate of aperture-integrated speckles. By a straightforward extension of the derivation of the level crossing rate for a normal distribution, Yura and McKinley derived the corresponding rate for the log-normal distribution [13], and while making a simplifying assumption, an integral expression for the level crossing rate for the gamma-gamma distribution has been obtained by Vetelino et al. [14].

In general, however, the level crossing rate for an arbitrary random process has not been found. Thus, for example, closed-form analytic results for the mean level crossing rate for a uniform distribution, a power law distribution, and the gamma-gamma distribution are unavailable. This paper will introduce a novel method for deriving closed-form analytical expressions for the mean level crossings for signals obeying an arbitrary probability density function (PDF) and in particular for various PDFs for signals of interest to the optical community. In general, the analytical results for the mean level crossing rate are supported and confirmed by numerical simulations. In particular, we illustrate the present method by presenting analytic expressions for $\nu\left(x_{0}\right)$ that previously were unavailable (including those mentioned above), and these results are confirmed by numerical simulations. However, as discussed below, in a limited number of cases the present results differ somewhat from the result obtained originally by Khimenko (and subsequently by others). This discrepancy remains unexplained and is laid open for future discussion.

The essence of the present method is the introduction of a unique bijective mathematical transformation that converts a signal obeying an arbitrary PDF into a signal that obeys a normal distribution. This allows one, as discussed below, to use the mean level crossing rate for the normal distribution to obtain the corresponding result for an arbitrary PDF. In Section 2 this transformation is introduced and discussed, and a general expression for the mean level crossing rate for and arbitrary PDF is derived. In Section 3 new results for several PDFs of interest are presented, compared with numerical simulations, and discussed. Additionally, as alluded to above, the deviations of the present analysis with some previously published results, which are not presently understood, are presented and laid open for discussion. However, despite these discrepancies we feel that the present work represents a step forward in our understanding of the level crossing rate for arbitrary random processes and should be made available in the literature. Finally, in Section 4 we present our concluding remarks.

\section{GENERAL CONSIDERATIONS}

Here we consider only differentiable stationary random processes (i.e., random processes that are continuous in the mean square sense) designated by " $X$ " and " $Y$." The $X$ process is assumed known in that all the various probability functions are implicitly given to us, and we would like to find out the corresponding functions of the $Y$ process. In particular, we would like to obtain the joint probability density function of $y$ and its time derivative, given the corresponding joint probability function of the $X$ process. In the following we denote by $x(t)$ and $y(t)$ the values of the random process $X$ and $Y$ at time $t$, respectively.

Let $p_{X}(x)$ and $p_{Y}(y)$ be two arbitrary PDFs. Consider a transformation of variables given by

$$
x=f(y),
$$

where the arbitrary (real) function $f$ is bijective (i.e., a single value of $y$ corresponds to a single value of $x$ and vice versa). By the standard laws of transformation of PDFs we have

$$
p_{Y}(y)=\left|f^{\prime}(y)\right| p_{X}[x(y)] \quad\left(\text { where } f^{\prime}(y) \equiv \frac{d f(y)}{d y}\right) .
$$

Next consider the joint PDF of the random variable $y$ and its first derivative with respect to time $\dot{y}, p_{Y \dot{Y}}(y, \dot{y})$.

Note that from Eq. (3) it follows directly from the chain rule of differentiation that

$$
\dot{x}=\frac{d f(y)}{d y} \frac{d y}{d t}=f^{\prime}(y) \dot{y} .
$$

Here we assume that $x$ is not a function of $\dot{y}$ and thus the joint $\mathrm{PDF}, p_{Y \dot{Y}}(y, \dot{y})$, is related to the corresponding joint PDF of $x$ and $\dot{x}$ by the Jacobian $[15,16]$ :

$$
\begin{aligned}
p_{Y \dot{Y}}(y, \dot{y})= & p_{X \dot{X}}(x[y], \dot{x}[y, \dot{y}])\left|\begin{array}{cc}
\frac{\partial x}{\partial y} & \frac{\partial x}{\partial \dot{y}} \\
\frac{\partial \dot{x}}{\partial y} & \frac{\partial \dot{x}}{\partial \dot{y}}
\end{array}\right|=p_{X \dot{X}}(x[y], \dot{x}[y, \dot{y}]) \\
& \times\left|\begin{array}{cc}
f^{\prime}(y) & 0 \\
f^{\prime \prime} \dot{y} & f^{\prime}(y)
\end{array}\right|=\left[f^{\prime}(y)\right]^{2} p_{X \dot{X}}(x[y], \dot{x}[y, \dot{y}]) .
\end{aligned}
$$

This is true for any bijective transformation of Eq. (3) that is not a function of the time derivative. Equation (6) relates the joint PDF of the random process of interest " $Y$ " to that of the corresponding PDF of the assumed known process " $X$."

Now consider the special case where $X$ is a zero-mean, unit-variance, normally distributed random process. It is well known that for a normally distributed random variable the joint PDF $p_{X X}(x, \dot{x})$ is given by (i.e., a normal stationary random process and its derivative are independent [17])

$$
p_{X \dot{X}}(x, \dot{x})=p_{X}(x) p_{\dot{X}}(\dot{x}),
$$

where $p_{X}(x)=\exp \left[-x^{2} / 2\right] / \sqrt{2 \pi}, p_{\dot{X}}(\dot{x})$ is also of Gaussian form given by [17] 


$$
p_{\dot{X}}(\dot{x})=\frac{1}{\sqrt{2 \pi \sigma_{\dot{x}}^{2}}} \exp \left[-\frac{\dot{x}^{2}}{2 \sigma_{\dot{x}}^{2}}\right],
$$

and $\sigma_{\dot{x}}^{2}$ is the variance of the time derivative of $x$. For the zero-mean, unit-variance normal PDF, we obtain from Eqs. (7) and (8)

$$
\begin{aligned}
p_{Y \dot{Y}}(y, \dot{y})= & f^{\prime}(y) \frac{1}{\sqrt{2 \pi}} \exp \left[-\frac{f^{2}(y)}{2}\right] \times f^{\prime}(y) \frac{1}{\sqrt{2 \pi \sigma_{\dot{x}}^{2}}} \\
& \times \exp \left[-\frac{f^{\prime 2}(y) \dot{y}^{2}}{2 \sigma_{\dot{x}}^{2}}\right]=p_{Y}(y) \frac{f^{\prime}(y)}{\sqrt{2 \pi \sigma_{\dot{x}}^{2}}} \\
& \times \exp \left[-\frac{f^{\prime 2}(y) \dot{y}^{2}}{2 \sigma_{\dot{x}}^{2}}\right] .
\end{aligned}
$$

Next, we express $\sigma_{\dot{x}}^{2}$ in terms of parameters related to the random process of interest. To do this we note that the mean value of the time derivative of any stationary random process is zero [18], from which it follows that the unconditional variance of the time derivative of the $Y$ process is given by

$$
\sigma_{\dot{y}}^{2}=\iint d y d \dot{y} \dot{y}^{2}(y) p_{Y \dot{Y}}(y, \dot{y}) .
$$

Substituting Eq. (9) into Eq. (10) and performing the integration over $\dot{y}$, simplifying, and rearranging terms yields

$$
\sigma_{\dot{x}}^{2}=\sigma_{\dot{y}}^{2} / \gamma,
$$

where the constant $\gamma$ (which is independent of the level) is given by

$$
\gamma=\int d y \frac{p_{Y}(y)}{f^{\prime 2}(y)} .
$$

Equation (11) gives the relationship between the variance of the derivative of the zero-mean, unit-variance normal distribution and the variance of the derivative of the random process of interest. Thus, Eq. (9), with $\sigma_{\dot{x}}^{2}$ given by Eq. (11), depends only on parameters related to the random process of interest. We note for any stationary random process that

$$
\sigma_{\dot{y}}^{2}=-\left.\ddot{R}_{Y}(\tau)\right|_{\tau=0}=\frac{\int_{0}^{\infty} d \omega \omega^{2} S_{Y}(\omega)}{\int_{0}^{\infty} d \omega S_{Y}(\omega)},
$$

where $R_{Y}(\tau)$ is the temporal autocorrelation coefficient of the random process of interest and $S_{Y}(\omega)$ is the corresponding temporal power spectrum.

In general, for an arbitrary transformation function $f(y)$, one does not obtain a normal process $x$. We will show that the transformation of variables given by

$$
x=\sqrt{2} \operatorname{erf}^{-1}\left[2 F_{Y}(y)-1\right],
$$

where $F_{Y}(y)$ is the cumulative distribution function (CDF) of the arbitrary stationary random process characterized by the $\operatorname{PDF} p_{Y}(y)$, yields a zero-mean, unit-variance normally distributed random variable $x$. The transformation given by Eq. (14) is bijective (i.e., one-to-one) and thus the transformed PDF, $p_{X}(x)$, is given by (see [15], Sec. 5-2, pp. 125-126)

$$
p_{X}(x)=\frac{p_{Y}[y(x)]}{\left|\frac{d x}{d y}\right|}
$$

From Eq. (14) we obtain

$$
\begin{aligned}
\frac{d x}{d y} & =\sqrt{2 \pi} \exp \left[\left(\operatorname{erf}^{-1}\left[2 F_{Y}(y)-1\right]\right)^{2}\right] \frac{d F_{Y}[y(x)]}{d y} \\
& =\sqrt{2 \pi} \exp \left[\left(\operatorname{erf}^{-1}\left[2 F_{Y}(y)-1\right]\right)^{2}\right] p_{Y}[y(x)],
\end{aligned}
$$

where $p_{Y}(y)$ is the corresponding PDF of the arbitrary distribution. Substituting Eq. (16) into Eq. (15) and simplifying yields

$$
\begin{aligned}
p_{X}(x) & =\frac{p_{Y}[y(x)]}{\sqrt{2 \pi} \exp \left[\left(\operatorname{erf}^{-1}\left[2 F_{Y}[y(x)]-1\right]\right)^{2}\right] p_{Y}[y(x)]} \\
& =\frac{1}{\sqrt{2 \pi} \exp \left[\left(\operatorname{erf}^{-1}\left[2 F_{Y}[y(x)]-1\right]\right)^{2}\right]} .
\end{aligned}
$$

Now from Eq. (14) we obtain that $2 F_{Y}[y(x)]-1=\operatorname{erf}[x / \sqrt{2}]$, from which it follows that

$$
p_{X}(x)=\frac{\exp \left[-x^{2} / 2\right]}{\sqrt{2 \pi}}, \quad \text { QED. }
$$

Thus, the transformation given by Eq. (14) transforms the arbitrary random process of interest into a zero-mean, unit-variance normal process, and hence Eq. (9) is the joint PDF of an arbitrary random process and its time derivative. Each level " $y$ " is uniquely mapped onto a single normally distributed level " $x$." Hence the mean level crossing rate for the $Y$ process can be obtained from Rice's results for the zero-mean, unit-variance normal process. The mean level crossings at level $x_{0}$ (i.e., for the sum of both positive and negative crossings) for a zero-mean, unit-variance normal process, as obtained from Eq. (2), is given by

$$
\nu\left(x_{0}\right)=\frac{\sqrt{\sigma_{\dot{x}}^{2}}}{\pi} \exp \left(-\frac{x_{0}{ }^{2}}{2}\right) .
$$

Substituting Eq. (14) into Eq. (19) [or, equivalently, substituting Eq. (14) into Eq. (1) and performing the integration over $\dot{y}]$ yields that the mean level crossing rate for an arbitrary random differentiable process is given by

$$
\nu\left(y_{0}\right)=\frac{\sigma_{\dot{x}}}{\pi} \exp \left[-f^{2}\left(y_{0}\right) / 2\right]=\frac{\sigma_{\dot{y}}}{\pi \sqrt{\gamma}} \exp \left[-f^{2}\left(y_{0}\right) / 2\right],
$$

where $\gamma$ and $\sigma_{\dot{y}}$ are obtained from Eqs. (12) and (13), respectively, and 


$$
x_{0}=f\left(y_{0}\right)=\sqrt{2} \operatorname{erf}^{-1}\left[2 F_{Y}\left(y_{0}\right)-1\right] .
$$

This is the main result of this paper. For a given analytical representation of the $\mathrm{CDF}$, the corresponding mean level crossing rate is given by Eq. (20). If, however, the $\mathrm{CDF}$ is not available in analytic form, numerical results can be obtained readily from the corresponding integral form of the CDF. Examination of Eq. (20) reveals that, in general, the maximum value of the mean level crossing rate occurs at the level $y_{\max }$ that satisfies $F_{Y}\left(y_{\max }\right)=1 / 2$.

Note that $\sigma_{\dot{y}}=\sqrt{\int_{0}^{\infty} d \omega \omega^{2} S_{Y}(\omega) / \int_{0}^{\infty} d \omega S_{Y}(\omega)}$ is independent of the level; rather, it depends on the specific physical circumstance of the problem at hand and represents a relative measure of the absolute magnitude of the mean level crossing rate. For example, the clear-air turbulenceinduced scintillation power spectrum for slant range propagation through the atmosphere is a function of both the zenith angle and the direction and magnitude of the normal component of the "effective" wind speed with respect to the line of sight. As a result, in the following, we focus our attention on the profile shape function of the mean level crossing rate, given by the exponential on the right-hand side of Eq. (20), and, in the following figures, we normalize the maximum value of $\nu\left(y_{0}\right)$ to unity.

In general, the mean frequency of negative crossings, $\nu^{-}$, must equal the mean frequency of positive crossing, $\nu^{+}$, so that $\nu=\nu^{+}+\nu^{-}=2 \nu^{+}=2 \nu^{-}$. Thus, for example, the mean duration of a fade at level $y_{0}, T^{-}\left(y_{0}\right)$ is

$$
T^{-}\left(y_{0}\right)=\frac{F_{Y}\left(y_{0}\right)}{\nu^{-}\left(y_{0}\right)},
$$

while the corresponding duration of a surge, $T^{+}\left(y_{0}\right)$, is

$$
T^{+}\left(y_{0}\right)=\frac{1-F_{Y}\left(y_{0}\right)}{\nu^{+}\left(y_{0}\right)} .
$$

The crossing level for which the mean duration of a fade equals the mean duration of a surge occurs for $F_{Y}\left(y_{0}\right)$ $=1 / 2$.

\section{ILLUSTRATIVE EXAMPLES AND COMPARISON TO PREVIOUS WORK}

In what follows, as appropriate, we normalized the level either to its mean or otherwise, as noted.

\section{A. Log-Normal Distribution}

The log-normal PDF and CDF are given by

$$
p_{Y}(y)=\left\{\begin{array}{ll}
\frac{\exp \left[-\frac{(\log y-\mu)^{2}}{2 \sigma^{2}}\right]}{\sqrt{2 \pi} \sigma y}, & \text { for } y \geqslant 0 \\
0 & \text { otherwise }
\end{array},\right.
$$

where "log" denotes the natural logarithm, $\mu$ and $\sigma$ are the mean and standard deviation of $\log y$, respectively, and

$$
F_{Y}(y)=\frac{1}{2}\left(1+\operatorname{erf}\left[\frac{\log y-\mu}{\sqrt{2} \sigma}\right]\right) .
$$

Substituting Eq. (25) into Eq. (20) and simplifying yields that the profile shape for the log-normal distribution is given by

$$
\nu\left(y_{0}\right)=\text { constant } \times \exp \left[-\frac{\left(\log y_{0}-\mu\right)^{2}}{2 \sigma^{2}}\right],
$$

in agreement with the results of [13].

Next, we illustrate the usefulness of Eq. (18) by presenting the mean level crossing rate for a number of PDFs that have not been previously obtained and compared these results with numerical simulations. The CDF of an arbitrary random process is a bijective monotonically increasing function of its argument. Consider two random processes specified by CDFs $F_{X}(x)$ and $F_{Y}(y)$. Now for any given value of $x$, the transformation $y=F_{Y}^{-1}\left[F_{X}(x)\right]$ yields a single unique value of $y$ that satisfies $F_{Y}(y)=F_{X}(x)$. In particular, a discrete random sample set $x_{j}$ is mapped onto a corresponding set $y_{j}$ whose underlying statistics are given by $p_{Y}(y)$. In particular, the analytic results are compared with corresponding numerical simulations of discrete correlated signal samples, as such samples are generally obtained in practice. The method used here to obtain correlated signal samples for an arbitrary random process $Y$ is as follows. Let $x$ be a zero-mean, unit-variance normally distributed random variable. Then, because a normal process plays such a central role in obtaining correlated simulation samples, we show explicitly in Appendix A that $F_{Y}^{-1}\left[F_{X}(x)\right]$ has the PDF of interest $p_{Y}$. Correlated normal signal samples for a zero-mean, unit-variance normal process are readily obtained using the UhlenbeckOrnstein method of producing a normally distributed signal $x$ with an arbitrary degree of correlation [19]. This inverse method can be used when the inverse function $F_{Y}^{-1}$ can be obtained explicitly or can be accurately approximated. For each of the comparison cases shown below, more than $10^{5}$ samples have been employed.

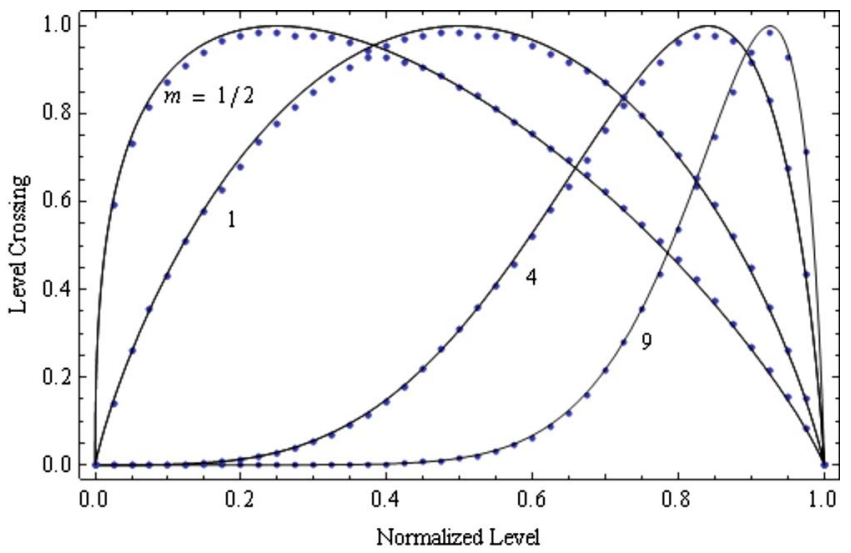

Fig. 1. (Color online) Relative level crossing rate for the power law PDF for various values of $m$. The analytic and simulation results are given by the solid and dotted curves, respectively. 


\section{B. Power Law Distribution}

The PDF is given by

$$
p_{Y}(y)=\left\{\begin{array}{ll}
m y^{m-1}, & \text { for } 0 \leqslant y \leqslant 1 \\
0, & \text { otherwise }
\end{array},\right.
$$

where $m$ is an arbitrary real number greater than zero. For example, such a PDF describes the irradiance statistics for a Gaussian-shaped laser beam in the presence of line-of-sight mechanical platform jitter, where $m$ is given by the square of the ratio of the $1 / \sqrt{e}$ angular beam radius to the 1- $\sigma$, single-axis standard deviation of jitter [20]. For this PDF we have

$$
F_{Y}(y)=y^{m} .
$$

Figure 1 is a plot of the relative level crossing rate as a function of normalized level for various values of $m$. Examination of Fig. 1 reveals that excellent agreement is obtained between the analytic and the simulation results. Note that the special case $m=1$ corresponds to a uniform PDF between zero and unity, and hence the $m=1$ curve
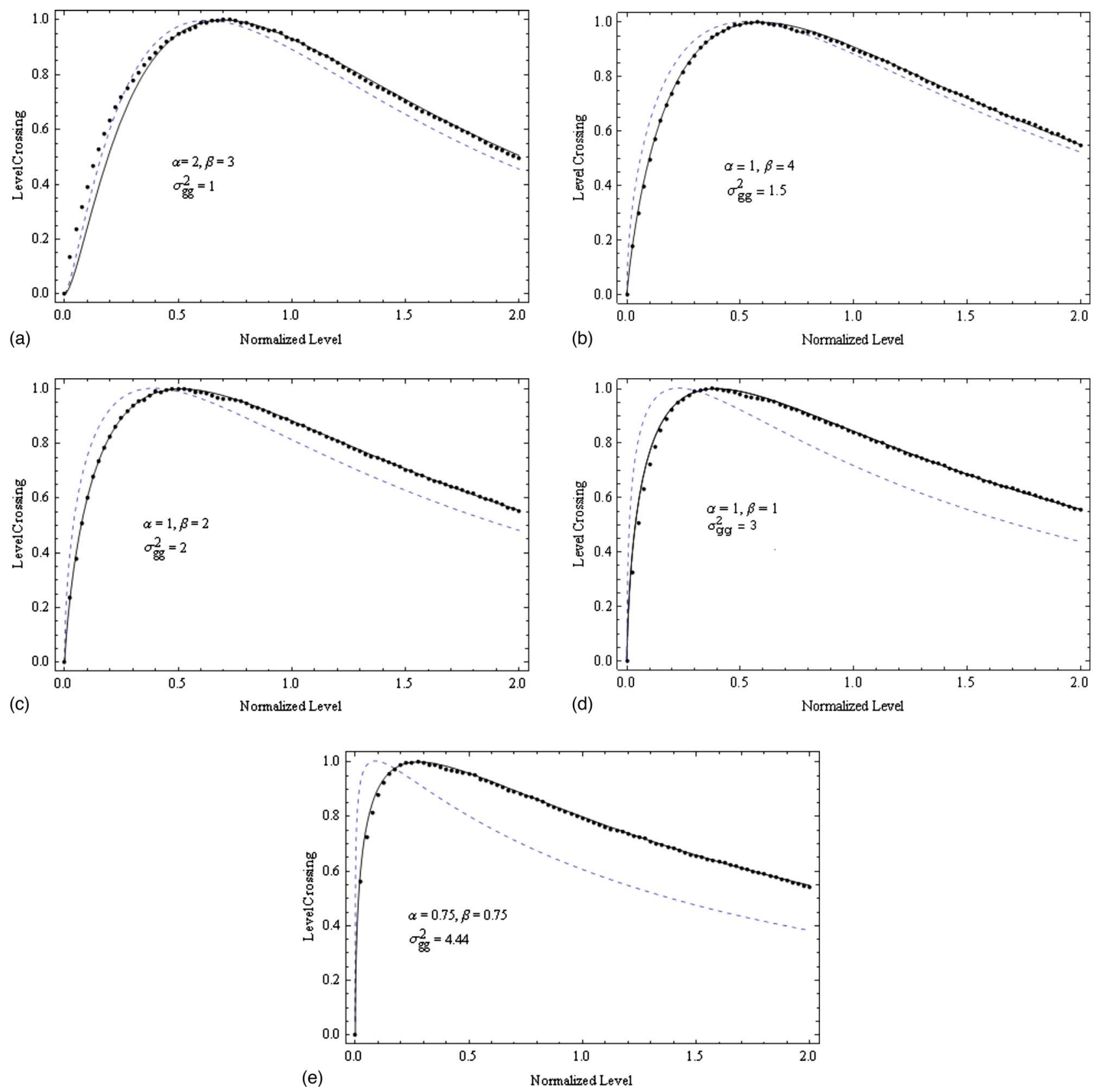

Fig. 2. (Color online) Mean level crossing rate for the gamma-gamma distribution as a function of the level for various values of $\alpha$ and $\beta$. The solid curve is the analytic result, the dashed curve is the "new integral expression" obtained in [14], and the points are our simulation results, which are obtained from a very accurate inversion approximation to Eq. (30). 
represents the mean level crossing rate for the uniform distribution.

\section{Gamma-Gamma Distribution}

The PDF of the gamma-gamma distribution is given by $[21,14]$

$$
p_{Y}(y)=\frac{2(\alpha \beta)^{(\alpha+\beta) / 2}}{\Gamma(\alpha) \Gamma(\beta)} y^{(\alpha+\beta) / 2-1} K_{\alpha-\beta}(2 \sqrt{\alpha \beta y}), \quad \text { for } y \geqslant 0,
$$

where $K_{n}(\cdot)$ is the modified Bessel function of the second kind of order $n$, and $\alpha$ and $\beta$ are free parameters. The corresponding $\mathrm{CDF}$ is

$$
\begin{aligned}
F_{Y}(y)= & \frac{\pi \csc [\pi(\beta-\alpha)]}{\Gamma(\alpha) \Gamma(\beta)} \\
& \times\left[\begin{array}{c}
\Gamma(\alpha)(\alpha \beta y)^{\alpha}{ }_{1} F_{2}(\alpha ; 1+\alpha, 1+\alpha-\beta ; \alpha \beta y) \\
-\Gamma(\beta)(\alpha \beta y){ }_{1}{ }_{1} F_{2}(\beta ; 1+\beta, 1-\alpha+\beta ; \alpha \beta y)
\end{array}\right],
\end{aligned}
$$

where $\Gamma(\cdot)$ is the gamma function and ${ }_{1} F_{2}$ denotes a generalized hypergeometric function [22]. It can be readily verified that the mean of this distribution is unity and the variance is given by

$$
\sigma_{g g}^{2}=\frac{1}{\alpha}+\frac{1}{\beta}+\frac{1}{\alpha \beta}
$$

The gamma-gamma distribution has been used to model the irradiance distribution for moderate-to-strong clearair turbulence-induced scintillation in the atmosphere, where $\sigma_{g g}^{2}>1$ [23]. The resulting mean level crossing rate, obtained from Eqs. (30) and (20), is plotted in Figs. 2 (a)-2(e) as a function of the level for various values of $\alpha$ and $\beta$ consistent with a variance $\geqslant 1$. The solid curve is the analytic result, the dashed curve is the "new integral expression" obtained in [14], and the points are our simulation results, which are obtained from a very accurate inversion approximation to Eq. (30).

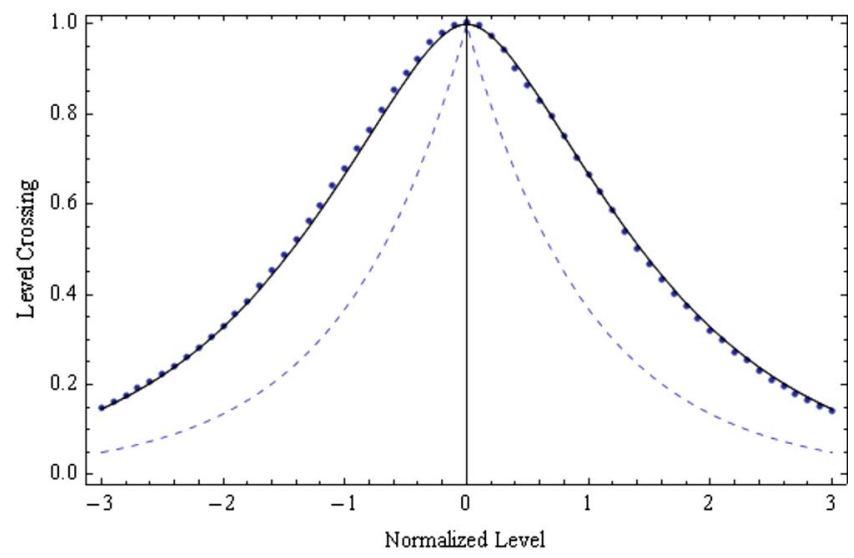

Fig. 3. (Color online) Mean level crossing rate for the Laplace distribution as a function of normalized level. The solid curve and the dotted curve are the new analytic results and the simulation, respectively, while the dashed curve is the analytic result of Khimneko.
Examination of Figs. 2(a)-2(e) reveals very good agreement between the analytic theory and the numerical simulations. In contrast, the "new integral expression" for the mean level crossing rate, which is based on an unjustified simplification [see the paragraph below Eq. (21) of [14]] is less accurate, and this inaccuracy increases with increasing values of the variance.

\section{Laplace Distribution}

The statistics of the intensity difference obtained from two small separated apertures is described by the Laplace distribution [24]. For simplicity, consider a Laplace distribution with zero mean and a scale parameter of unity. The PDF and CDF of this distribution are given by [22]

$$
p_{Y}(y)=\frac{1}{2} \exp (-|y|) \quad \text { for }-\infty \leqslant y \leqslant \infty
$$

and

$$
F_{Y}(y)=\left\{\begin{array}{ll}
\frac{e^{y}}{2}, & y \leqslant 0 \\
1-\frac{e^{-y}}{2}, & y \geqslant 0
\end{array} .\right.
$$

In Fig. 3 the mean level crossing rate as a function of the level (normalized here to unity) is compared with both simulation and the analytic results of Khimenko. Examination of Fig. 3 reveals excellent agreement between our "new" analytic results, based on Eqs. (33) and (20). In contrast, Khimenko's corresponding result of $\nu_{\text {Khimenko }}\left(y_{0}\right)$ $=$ constant $\times \exp \left(-\left|y_{0}\right|\right)$ is inaccurate.

\section{E. Weibull Distribution}

For simplicity we consider a Weibull distribution with shape parameter $k$ and a scale parameter of unity. The $\mathrm{PDF}$ and CDF of this distribution is given by [22]

$$
p_{Y}(y)=k y^{k-1} \exp \left[-y^{k}\right], \quad y \geqslant 0,
$$

and

$$
F_{Y}(y)=1-\exp \left[-y^{k}\right]
$$

where $k$ is a real number greater than zero. For example, this distribution is used to model wind speeds at a given site. Note that for $k=1$ and 2 the Weibull distribution reduces to the exponential and the Rayleigh distribution, respectively. In addition to the general inverse $\mathrm{CDF}$ method, discussed in Appendix A, of simulating correlated discrete samples, there exists for the Weibull distribution an alternative method for doing the same. As shown in Appendix B, the transformation of variables

$$
z=\left(\frac{x_{1}^{2}+x_{2}^{2}}{2}\right)^{1 / k}
$$

where $x_{1}$ and $x_{2}$ are zero-mean, unit-variance independent normally distributed random variables and $k$ is a real number greater than zero, yields the Weibull distribution. In the following we refer to this as the "sum of squares" method. In Figs. 4(a)-4(e) the mean level crossing rate is plotted versus the level, (normalized to unity) for various values of $k$ and compared with both simulation 

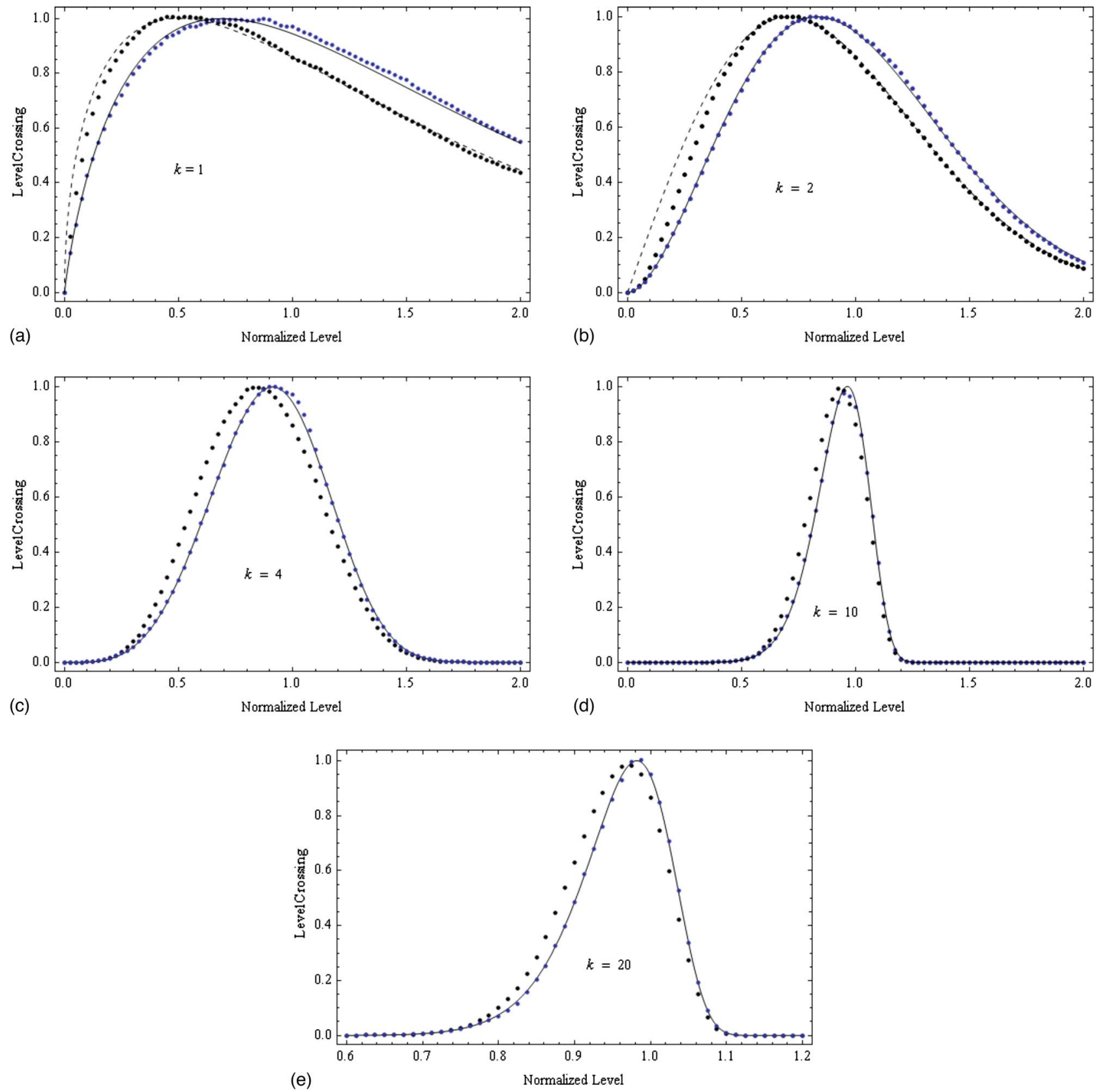

Fig. 4. (Color online) Mean level crossing rate for the Weibull distribution as a function of normalized level for various values of the shape parameter $k$. In each panel the right-hand solid and dotted curves are our analytic result and simulation results, respectively, based on the inverse CDF method, while the left-hand dotted black curves and dashed curves (for $k=1$ and 2 ) are the simulation results based on the sum of squares method and Khimenko's analytic results (for $k=1, \nu\left(y_{0}\right) \propto y_{0}^{1 / 2} e^{-y_{0}}$, and for $\left.k=2, \nu\left(y_{0}\right) \propto y_{0} e^{-y_{0}^{2} / 2}\right)$, respectively.

methods and for the exponential and Rayleigh distribution with Khimenko's analytic results. In each of Figs. 4 the right-hand solid and dotted curves are our analytic result and simulation results, respectively, based on the inverse CDF method, while the left-hand dotted black curves and dashed curves (for $k=1$ and 2) are the simulation results based on the sum of squares method and Khimenko's analytic results (for $k=1, \nu\left(y_{0}\right) \propto y_{0}^{1 / 2} e^{-y_{0}}$ and for $\left.k=2, \nu\left(y_{0}\right) \propto y_{0} e^{-y_{0}^{2} / 2}\right)$, respectively.

Examination of Fig. 4 reveal that for $k$ of the order unity our analytic results and the simulations based on the inverse $\mathrm{CDF}$ method do not agree with the analytic results of Khimenko for $k=1$ and 2 and simulations based on the sum of squares method, while for larger values of $k$ the simulation-based on the sum of squares method tend to coalesce both to the present analytical theory and to simulations based on the inverse CDF method. The discrepancy for small $k$ values is reduced as the $k$ number increases, which is accompanied by the PDF being less discontinuous at zero signal level. Thus, this discrepancy could have either of two causes. The first possibity is a numerical instability, as the transformation from, e.g., an exponential $(k=1)$ to a normal PDF will call for the majority of occurrences of exponentially distributed signal level near zero being transformed toward large negative values in the normally distributed regime. A second rea- 

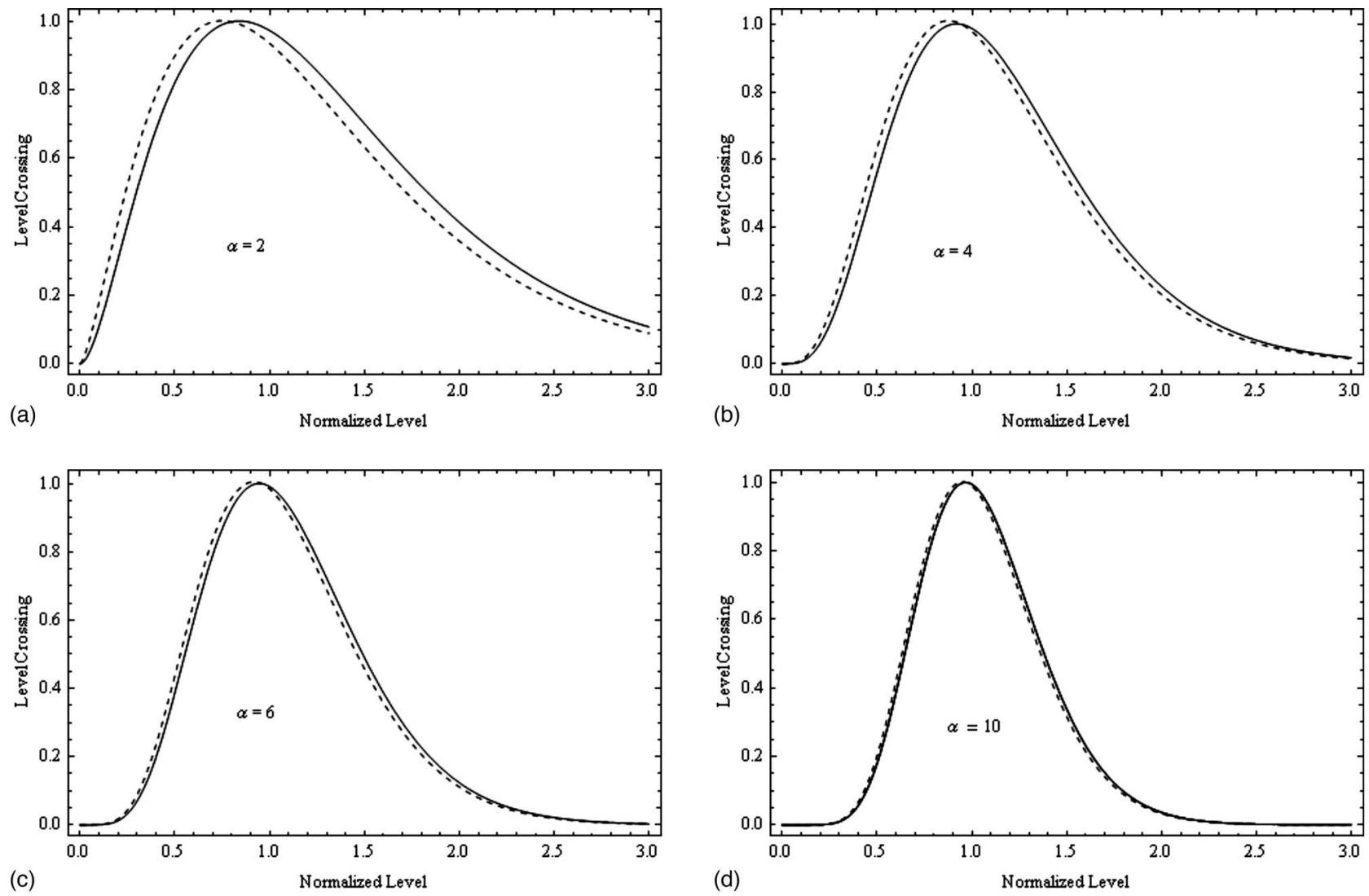

Fig. 5. Mean level crossing rate for the gamma distribution as a function of level normalized to the mean. In each panel the solid and dashed curves are our analytic results and the corresponding results of Barakat, given by Eq. (4.2) of [4], respectively.

son for the discrepancy could be that simulations have shown that the level crossing rate depends on the correlation time versus the sampling time interval. Simulations with signals derived by different numerical methods have even yielded different results for the level crossings, yet their statistics yield virtually identical moments, which are in accordance with textbook values. The reason for this discrepancy, however, is currently not understood.

\section{F. Gamma Distribution}

Next, we consider the gamma distribution, which has been used extensively in the literature to model the PDF of aperture-integrated speckle $[4,10]$. This PDF and corresponding $\mathrm{CDF}$ are given by

$$
p_{Y}(y)=\frac{1}{\Gamma(\alpha)} \alpha^{\alpha} y^{\alpha-1} \exp [-\alpha y], \quad \text { for } y \geqslant 0
$$

and

$$
F_{Y}(y)=1-\frac{\Gamma(\alpha, \alpha y)}{\Gamma(\alpha)},
$$

where for simplicity in notation we have normalized the level, $y$, to its mean and $\Gamma(\alpha, \alpha y)$ is the incomplete gamma function. The parameter $\alpha$ can be physically interpreted as the mean number of speckles contained within a collecting aperture [10]. Figures 5(a)-5(d) compare the present analytic results (solid curves) for the mean level crossing rate with the corresponding results obtained by Barakat [Eq. (4.2)] [4] (dashed curves) for various values of $\alpha$. Because for $\alpha=1$ the PDF of the gamma distribution becomes the exponential PDF, we omit this case from Fig. 5 , as it has been considered previously.

We remark that simulations of the mean level crossing rate for the gamma distribution based on the inverse CDF method are in excellent agreement with our analytical results, and therefore, for presentation purposes they are not shown in Fig. 5. Examination of Fig. 5 reveals that for $\alpha$ less than about 4 our analytic results are somewhat different from the corresponding results of Barakat [4], while for larger values of $\alpha$ the two results are in very good agreement. Again, the reason for this discrepancy for small values of $\alpha$ is currently not understood.

\section{G. Rice-Nakagami Distribution}

As a final example we illustrate the utility of obtaining the mean level crossing rate for a PDF whose corresponding CDF cannot be obtained analytically [25]. The PDF and CDF of the Rice-Nakagami is given by

$$
p_{Y}(y)=2 y \exp \left[-\left(y^{2}+C^{2}\right)\right] I_{0}(2 y C), \quad \text { for } y \geqslant 0
$$

and

$$
F_{Y}(y)=\int_{0}^{y} 2 x \exp \left[-\left(x^{2}+C^{2}\right)\right] I_{0}(2 x C) d x,
$$

where $I_{0}(\cdot)$ is the modified Bessel function of the first kind the order zero, $C$ is a real constant, and both $y$ and $C$ are normalized to twice the variance of the underlying normal distribution [26]. In a variety of applications, the 


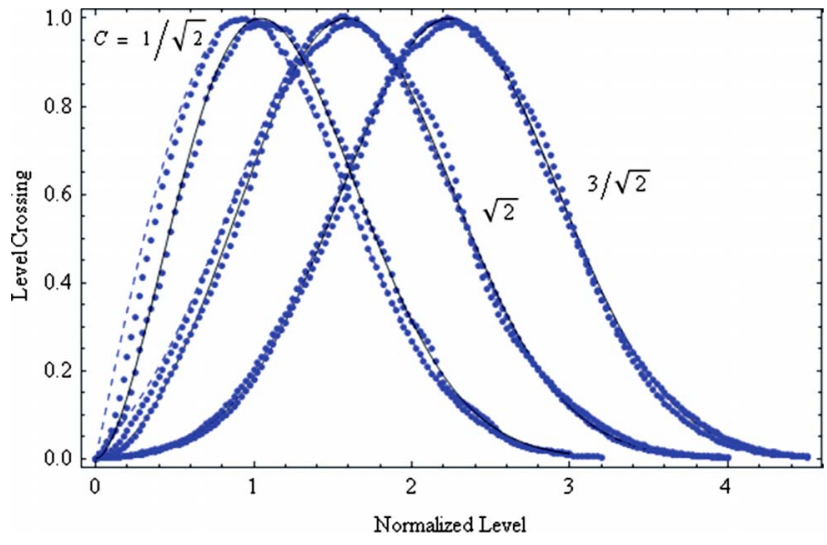

Fig. 6. (Color online) Mean number of (normalized) level crossings for the Rice-Nakagami distribution as a function of normalized level for various values of $C$. For each $C$ value the righthand solid curve and dotted curve are our analytic result and simulation results, respectively, based on the inverse CDF method, while the left-hand dotted black curve and dashed curve are the simulation results based on the above sum of squares method and a normalized version of Eq. (42), respectively.

Rice-Nakagami distribution is used to model the intensity distribution in a speckle pattern that consists of a specular component and a diffuse scattered component [27]. Rice has shown that the mean level crossing rate is directly proportional to the PDF and is given by [28]

$$
\nu\left(y_{0}\right)=\sqrt{\frac{-\ddot{R}(0)}{2 \pi}} p_{Y}\left(y_{0}\right) .
$$

In addition to the inverse CDF method of obtaining correlated sample simulations, one also can obtain such samples for the Rice-Nakagami distribution from an extension of the sum of squares method discussed in Appendix B given by

$$
y=\sqrt{\frac{\left(x_{1}-C\right)^{2}+x_{2}^{2}}{2}}
$$

where $x_{1,2}$ are independent, zero-mean, unit-variance normal distributions and $C$ is a real constant.

The mean number of (normalized) level crossings for the Rice-Nakagami distribution as a function of normalized level for various values of $C$ is plotted in Fig. 6 [29]. For each $C$ value the right-hand solid and dotted curve are our analytic result [i.e., obtained from Eq. (20)] and simulation results, respectively, based on the inverse CDF method, while the left-hand dotted black curve and dashed curve are the simulation results based on the above sum of squares method and a normalized version of Eq. (41), respectively. Because the inverse CDF function cannot be obtained explicitly for the Rice-Nakagami distribution, the corresponding inverse $\mathrm{CDF}$ simulations are obtained from an accurate curve fit to $F_{Y}^{-1}$. Similar to what was obtained for the Weibull and gamma distribution, examination of Fig. 6 reveals that for $C$ less than about 1.5-2 the results obtained from Eq. (20) and the inverse $C D F$ method are somewhat in disagreement with Rice's result and simulations based on the sum of squares method. However, for $C$ larger than about 2, both analytic and theoretical results are in very good agreement. As stated above, the reason for this discrepancy is not presently understood.

\section{CONCLUSION}

A method of transforming a series of random signals with known PDFs into a corresponding new series of random signals with a normal PDF has been obtained with the purpose of deriving the mean level crossing rate for the signal under consideration. The method is based on using known results for the mean level crossing rate for a normally distributed signal. The present results have been compared with numerical simulations derived by using an inverse transformation method based on having random, correlated samples of a normal process. These simulations have shown good agreement with the transformation method presented in this paper, although some discrepancies have been observed, the explanation of which has been laid open for discussion.

The following examples with relevance for the optics community have been analyzed:

- The log-normal distribution applicable for laser communication under weak scintillation conditions.

- The power-law distribution describing intensity variation for line-of-sight propagation with platform jitter.

- The gamma-gamma distribution previously presented as a model for intensity fluctuations under moderate-to-strong scintillation conditions.

- The Laplace distribution describing the distribution of intensity differences in speckle fields.

- The Weibull distribution used for describing distribution of wind velocities, here relevant for LIDAR systems.

- The Rice-Nakagami distribution applicable for irradiance conditions arising when a speckle field consists of a specular component and diffuse components or a diffused speckle field that is interfered with a deterministic local oscillator.

\section{APPENDIX A}

Consider the transformation of variables

$$
z=F_{Y}^{-1}\left[F_{X}(x)\right],
$$

where $X$ is a zero-mean, unit-variance normal random process. Inverting Eq. (A1) for the zero-mean, unitvariance normal distribution yields

$$
x=F_{X}^{-1}\left[F_{Y}(z)\right]=\sqrt{2} \operatorname{erf}^{-1}\left[2 F_{Y}(z)-1\right],
$$

where $\operatorname{erf}^{-1}(\cdot)$ denotes the inverse error function. We will now show that the PDF of the $Z$ process is $p_{Y}$, the PDF of the process of interest. Because we are dealing with bijective functions, the PDF of the transformed random variable is given by

$$
p_{Z}=p_{X}[x(z)]\left|\frac{d x}{d z}\right| \text {. }
$$

We have 


$$
p_{X}[x(z)]=\exp \left[-\left(\operatorname{erf}^{-1}\left[2 F_{Y}(z)-1\right]\right)^{2}\right],
$$

and from Eq. (A2)

$$
\begin{aligned}
\frac{d x}{d z} & =\sqrt{2 \pi} \exp \left[\left(\operatorname{erf}^{-1}\left[2 F_{Y}(z)-1\right]\right)^{2}\right] F_{Y}^{\prime}(z) \\
& =\sqrt{2 \pi} \exp \left[\left(\operatorname{erf}^{-1}\left[2 F_{Y}(z)-1\right]\right)^{2}\right] p_{Y}(z) .
\end{aligned}
$$

Substituting Eqs. (A4) and (A5) into Eq. (A3) yields $p_{Z}$ $=p_{Y}$.

QED.

Our simulation of correlated samples for an arbitrary $\mathrm{PDF} p_{Y}$ is based on Eq. (A1) where a correlated zeromean, unit-variance normal sample distribution $x_{j}$ is obtained from [19]

$$
x_{j}=e^{-t_{0}} x_{j-1}+\sqrt{1-e^{-2 t}} x_{\text {new }}, \quad j=1,2, \ldots N_{S},
$$

where $x_{j} \equiv x\left(t_{j}\right), N_{S}$ is the number of samples, and for each $j$ the numerical value of $x_{\text {new }}$ is obtained from an independent zero-mean, unit-variance normal distribution (i.e., $x_{j-1}$ and $x_{\text {new }}$ are independent). Physically, $t_{0}$ can be interpreted as the ratio of the sampling time interval to the correlation time of the process. For $t_{0} \ll 1$ and $t_{0} \gg 1 \mathrm{Eq}$. (A6) yields correlated and uncorrelated samples, respectively. Here we consider only correlated samples, obtained from Eq. (A6) with $t_{0} \leqslant 0.1$, and $N_{S} \geqslant 10^{5}$. Then the simulated level crossing rate, $\nu_{\text {sim }}\left(y_{0}\right)$, is obtained from

$$
\nu_{\text {sim }}\left(y_{0}\right)=\sum_{j=1}^{N_{S^{-1}}} c_{j}
$$

where the count for the $j$ sample is

$$
c_{j}=\left\{\begin{array}{l}
1 \text { if either } y_{j}>y_{0} \text { and } y_{j+1}<y_{0} \\
\text { or } y_{j}<y_{0} \text { and } y_{j+1}>y_{0} \\
0 \text { otherwise }
\end{array} .\right.
$$

\section{APPENDIX B}

Consider the transformation of variables

$$
z=\left(\frac{x_{1}^{2}+x_{2}^{2}}{2}\right)^{1 / k}
$$

where $x_{1}$ and $x_{2}$ are zero-mean, unit-variance independent normally distributed random variables and $k$ is a real number greater than zero. We now show that the PDF of the $Z$ process is the Weibull distribution considered in the text. Because $x_{1}$ and $x_{2}$ are independent, we have that the PDF of Eq. (B1) is given by

$$
\begin{aligned}
p_{Z}(z) & =\int_{-\infty}^{\infty} d x_{1} \int_{-\infty}^{\infty} d x_{2} p_{X}\left(x_{1}, x_{2}\right) \delta\left(z-\left[\frac{x_{1}^{2}+x_{2}^{2}}{2}\right]^{1 / k}\right) \\
& =\int_{-\infty}^{\infty} d x_{1} \int_{-\infty}^{\infty} d x_{2} p_{X}\left(x_{1}\right) p_{X}\left(x_{2}\right) \delta\left(z-\left[\frac{x_{1}^{2}+x_{2}^{2}}{2}\right]^{1 / k}\right),
\end{aligned}
$$

where $p_{X}(x)=\exp \left[-x^{2} / 2\right] / \sqrt{2 \pi}$ and $\delta(\cdot)$ is the onedimensional Dirac delta function.

Substituting $p_{X}\left(x_{1}\right)$ and $p_{X}\left(x_{2}\right)$ into Eq. (B2), performing the integrations, and simplifying yields

$$
p_{Z}(z)=k z^{k-1} \exp \left[-z^{k}\right] \frac{1}{\pi} \int_{-\sqrt{2 z^{k}}}^{\sqrt{2 z^{k}}} \frac{d x_{1}}{\sqrt{2 z^{k}-x_{1}^{2}}}=k z^{k-1} \exp \left[-z^{k}\right]
$$

which is the Weibull distribution.

\section{ACKNOWLEDGMENTS}

We acknowledge financial support from the Danish Council for Technology and Innovation under the Innovation Consortium CINO (Centre for Industrial Nano Optics). The research of $\mathrm{H}$. T. Yura was performed while he was a guest scientist at DTU Fotonik, Denmark, SeptemberNovember 2009.

\section{REFERENCES}

1. S. O. Rice, "Mathematical analysis of random noise," in $\mathrm{Se}$ lected Papers on Noise and Stochastic Processes (Dover, Inc. New York, 1954).

2. P. Beckmann, Probability in Communication Engineering (Hartcourt Brace \& World, 1967), Sec. 6.7.

3. V. I. Khimenko, "The average number of trajectory overshoots of a non-Gaussian random process above a given level,” Izv. Vyssh. Uchebn. Zaved., Radiofiz. 21, 1170-1176 (1977).

4. R. Barakat, "Level-crossing statistics of aperture averagedintegrated isotropic speckle," J. Opt. Soc. Am. A 5, 1244 1247 (1988).

5. J. Bendat, Principles and Applications of Random Noise Theory (Wiley, 1958).

6. J. Abrahams, "A survey of recent progress on level crossing problems for random processes," in Communications and Networks, A Survey of Recent Advances, I. F. Blake and H. V. Poor, eds. (Springer-Verlag, 1986).

7. K. Ebeling, "Statistical properties of spatial derivatives of the amplitude and intensity of monochromatic speckle patterns," Opt. Acta 26, 1505-1521 (1979).

8. R. Barakat, "The level-crossing rate and above-level duration time of the intensity of a Gaussian random process," Inf. Sci. (N.Y.) 20, 83-87 (1980).

9. R. D. Bahuguna, K. K. Gupta, and K. Singh, "Expected number of intensity level crossings in a normal speckle pattern," J. Opt. Soc. Am. 70, 874-876 (1980).

10. J. W. Goodman, Speckle Phenomena in Optics: Theory and Applications (Roberts, 2006).

11. K. Eebeling, "Experimental investigation of some statistical properties of monochromatic speckle patterns," Opt. Acta 26, 1345-1349 (1979).

12. N. Takai, T. Iwai, and T. Asakura, "Real-time velocity measurements for a diffuse object using zero-crossings of laser speckle," J. Opt. Soc. Am. 70, 450-455 (1980).

13. H. T. Yura and W. G. McKinley, "Optical scintillation statistics for IR ground-to-space laser communication systems," Appl. Opt. 22, 3353-3358 (1983).

14. F. S. Vetelino, C. Young, and L. Andrews, "Fade statistics and aperture averaging for Gaussian waves in moderate-tostrong turbulence," Appl. Opt. 46, 3780-3789 (2007).

15. P. Beckmann, Probability in Communication Engineering (Hartcourt Brace \& World, 1967), Sec. 2.4, p. 73, Eq. 2.4-4.

16. A. Papoulis, Probability, Random Variables, and Stochastic Processes (McGraw-Hill, 1965), Sec. 7-2, pp. 201-202.

17. P. Beckmann, Probability in Communication Engineering (Hartcourt Brace \& World, 1967), Sec. 6.6, pp. 223 and 230.

18. P. Beckmann, Probability in Communication Engineering (Hartcourt Brace \& World, 1967), Sec. 6.6, Eq. 6.6-21, p. 222.

19. G. E. Uhlenbeck and L. S. Ornstein, "On the theory of Brownian motion," Phys. Rev. 36, 823-841 (1930).

20. H. T. Yura, "LADAR detection statistics in the presence of pointing errors," Appl. Opt. 33, 6482-6498 (1994). 
21. L. C. Andrews, R. L. Phillips, and C. Y. Hopen, Laser Beam Scintillation with Applications (SPIE Press, 2001), Sec. 2.5.

22. S. Wolfram, Mathematica, Version 7 (Cambridge Univ. Press, 2008).

23. See Ref. [14] and references therein.

24. S. G. Hanson and H. T. Yura, "Statistics of spatially integrated speckle intensity difference," J. Opt. Soc. Am. A 26, 371-375 (2009).

25. The $\mathrm{CDF}$ of the Rice-Nakagami distribution can be expressed in terms of the Marcum Q functions, which are tabulated, but not supported, to the best of our knowledge, by any commercial commuter programs such as Math- ematica and Matlab. Consequently, numerical integration of Eq. (3.17) is used to obtain the results indicated in Fig. 6.

26. P. Beckmann, Probability in Communication Engineering (Hartcourt Brace \& World, 1967), Sec. 4.4.

27. J. W. Goodman, Speckle Phenomena in Optics: Theory and Applications (Roberts, 2006), Sec. 3.2.2.

28. S. O. Rice, "Statistical properties of a sine wave plus random noise," Bell Syst. Tech. J. 27, 109-157 (1948).

29. Because for $\mathrm{C}=0$ the Rice-Nakagami distribution becomes the Rayleigh distribution, which has already been considered in Subsection 3.E, we omit this case in Fig. 6. 